\title{
Effects of SCFA on the DNA methylation pattern of adiponectin and resistin in high-fat-diet-induced obese male mice
}

\author{
Yuanyuan $\mathrm{Lu}^{1,2} \dagger$, Chaonan Fan ${ }^{1} \dagger$, Aimin Liang ${ }^{2}$, Xiuqin Fan ${ }^{1}$, Rui Wang ${ }^{1}$, Ping $\mathrm{Li}^{1}$ and Kemin $\mathrm{Qi}^{1 *}$ \\ ${ }^{1}$ Laboratory of Nutrition, Beijing Pediatric Research Institute, Beijing Children's Hospital, Capital Medical University, National \\ Center for Children's Health, Beijing 100045, People's Republic of China \\ ${ }^{2}$ Department of Child Health Care, Beijing Children's Hospital, Capital Medical University, National Center for Children's \\ Health, Beijing 100045, People's Republic of China
}

(Submitted 20 July 2017 - Final revision received 13 March 2018 - Accepted 25 April 2018 - First published online 21 June 2018 )

\section{Abstract}

Specific adipokines, such as adiponectin and resistin, are secreted from adipose tissue and are associated with the development of obesity. Supplementation of dietary SCFA can prevent and reverse high-fat-diet (HFD)-induced obesity. However, it is not clear whether SCFA ameliorate abnormal expression of adiponectin and resistin in the obese state. The aim of this study was to investigate the effects of SCFA on adiponectin and resistin's expressions in diet-induced obese mice, as well as the potential mechanisms associated with DNA methylation. C57BL/6J male mice were fed for 16 weeks with five types of HFD (34.9\% fat by wt., $60 \% \mathrm{~kJ}$ ) - a control HFD and four HFD with acetate (HFD-A), propionate (HFD-P), butyrate (HFD-B) and their admixture (HFD-SCFA). Meanwhile, a low-fat diet ( $4.3 \%$ fat by wt., $10 \% \mathrm{~kJ})$ was used as the control group. The reduced mRNA levels of adiponectin and resistin in the adipose tissue of the HFD-fed mice were significantly reversed by dietary supplementation of acetate, propionate, butyrate or their admixture to the HFD. Moreover, the expressional changes of adiponectin and resistin induced by SCFA were associated with alterations in DNA methylation at their promoters, which was mediated by reducing the expressions of enzyme-catalysed DNA methyltransferase (DNMT1, 3a, 3b) and the methyl-CpG-binding domain protein 2 (MBD2) and suppressing the binding of these enzymes to the promoters of adiponectin and resistin. Our results indicate that SCFA may correct aberrant expressions of adiponectin and resistin in obesity by epigenetic regulation.

\section{Key words: SCFA: Obesity: Adiponectin: Resistin: DNA methylation: Mice}

In the past decades, it has become apparent that dietary SCFA might have an important role in the prevention and treatment of obesity. Some epidemiological data indicate that SCFA reduce weight gain and body fat mass in obesity ${ }^{(1,2)}$. Subsequently, the study using rodent models of diet-induced obesity has further shown that supplementation of dietary SCFA prevents and reverses high-fat-diet (HFD)-induced metabolic abnormalities in mice ${ }^{(3)}$. SCFA, including acetate, propionate and butyrate, are the main products of the fermentation of dietary fibres by anaerobic intestinal microbiota ${ }^{(4)}$. In addition to serving as an energy source, SCFA are recognised as signalling molecules via G-protein-coupled receptors 41 and 43 (GPR41 and GPR43). SCFA bind to these receptors and activate G-protein-mediated second messenger signalling pathways, which have been identified to be involved in the modulation of lipid metabolism, in influencing leptin production, insulin sensitivity and other satiety hormones and in inhibiting lipolysis in adipose tissue ${ }^{(5-7)}$
Obesity is defined as an excessive growth of adipose tissue, which is also an active endocrine organ that is able to produce and release several bioactive polypeptides known as adipokines ${ }^{(8)}$. Adiponectin and resistin are major adipokines secreted by white adipose tissues, and their aberrant expressions may contribute to the progression of obesity and its complications including insulin resistance, inflammation, hypertension, cardiovascular risk and metabolic disorders ${ }^{(9)}$. It has been demonstrated that, in obesity, the adiponectin is expressionally reduced $^{(10)}$, whereas the resistin's expression is still in controversy with being up-regulated in initial studies and downregulated in later reports ${ }^{(11)}$. However, the mechanisms underlying the abnormal expression of these two adipokines in obesity are still far from being fully understood.

There is substantial evidence from animals and humans demonstrating that obesity predisposition and weight loss outcomes have been associated with changes in epigenetic patterns ${ }^{(12)}$. Methylation of cytosines within the CpG dinucleotide

Abbreviations: ChIP, chromatin immunoprecipitation; DIO, high-fat-diet-induced obese; DNMT, DNA methyltransferases; HFD, high-fat diet; HFD-A, HFD with acetate; HFD-B, HFD with butyrate; HFD-P, HFD with propionate; HFD-SCFA, HFD with admixture of acetate, butyrate and propionate; MBD2, methyl-CpGbinding domain protein 2 .

* Corresponding author: K. Qi, fax +86 10 59718700, email qikemin@bch.com.cn

$\dagger$ These authors contributed equally to this work. 
by DNA methyltransferases (DNMT) is involved in regulating transcription and chromatin structure, controlling transcriptional consequences of the gene. Moreover, the regional specificity of genomic DNA methylation patterning changes markedly with different environmental stimuli including diets ${ }^{(13)}$. Intriguingly, several reports have shown that the HFD consumption affects the epigenetics of various genes involved in the metabolic imbalance, adiposity, appetite regulation and so on ${ }^{(14)}$. It is also indicated that the obvious hypermethylation in the promoters of leptin and adiponectin were closely associated with its abnormal expression in obesity ${ }^{(15,16)}$.

More recently, nutrients and other environmental factors have been shown to induce epigenetic modifications, such as CPG island methylation and histone modifications, which, in turn, alter the gene expression, leading to susceptibility to many non-communicable chronic diseases ${ }^{(17,18)}$. SCFA are well known for their epigenetic activity as histone deacetylase inhibitors (HDACi), and activities of HDAC and methyl-CpGbinding domain protein (MBD), consequently, control gene expression in colon cancer cells and human embryonal kidney $293 \mathrm{M}+$ cell lines ${ }^{(19,20)}$. With regard to these considerations, we hypothesised that SCFA may ameliorate the development of obesity through influencing the DNA methylation levels in promoters of the adiponectin and resistin genes. Therefore, in this study, using the high-fat diet-induced obese (DIO) male mouse model $^{(21)}$, we determined the preventive effects of exogenous SCFA on body weight gain, and associated changes in expressions of the adiponectin and resistin and the underlying epigenetic mechanisms.

\section{Methods}

\section{Diets}

Five types of HFD (34.9\% fat by wt., $60 \% \mathrm{~kJ})$ - a control HFD and four HFD with acetate (HFD-A), propionate (HFD-P), butyrate (HFD-B) and their admixture (HFD-SCFA) - were designed according to the HFD formula (D12492) for the DIO mice from Research Diets, Inc. These five HFD contained the same amount of lard and soya oil as the main source of fat in each diet. Sodium acetate (W302406), sodium propionate (W518905), sodium butyrate (303410) (Sigma-Aldrich) or their admixture (at a ratio of 3:1:1) was incorporated into the HFD at a proportion of $5 \%$ of the diet $(\mathrm{w} / \mathrm{w})$. In addition, a low-fat diet ( $4.3 \%$ fat by wt., $10 \% \mathrm{~kJ}$ ) was also designed as a lean control with lard and soya oil as the source of fat, based on the control diet formula (D12450B) from Research Diets, Inc. (Table 1). The diets were prepared by the Institute of Laboratory Animal Sciences at the Chinese Academy of Medical Sciences and stored at $-20^{\circ} \mathrm{C}$ before use.

\section{Animals}

C57BL/6J male mice, 3-4 weeks old, purchased from the SPF Laboratory Animal Technology Co. Ltd, were randomly divided into six groups ( $n 10$ for each group) and housed at the animal facilities under a $12 \mathrm{~h}$ light- $12 \mathrm{~h}$ dark cycle with cycles of air ventilation and free access to water and food in the Laboratory Animal Center of the Academy of Military Medical Sciences of China. After 1 week of recovery from transportation, the mice were fed one of the five HFD for 16 weeks to induce obesity with the low-fat diet feeding as control, and the body weight was measured weekly. At the end of the experiments, mice were anaesthetised by intraperitoneal injection of Avertin $(125 \mathrm{mg} / \mathrm{kg}$ of 2,2,2-tribromoethanol, T-4840-2; Sigma-Aldrich, Chemie GmbH). After the mice were killed, the epididymal fat was dissected free of the surrounding tissue, removed, wrapped in $\mathrm{Al}$ foil and frozen in liquid $\mathrm{N}_{2}$ and transferred to $-80^{\circ} \mathrm{C}$ until the analysis was performed.

All of the animal experiments were performed in accordance with the recommendations in the Guide for the Care and

Table 1. Ingredient composition of the experimental diets fed to mice

\begin{tabular}{|c|c|c|c|c|c|c|}
\hline & Control & HFD & HFD-A & HFD-P & HFD-B & HFD-SCFA \\
\hline \multicolumn{7}{|l|}{ Ingredients (g/kg) } \\
\hline Casein & 200 & 260 & 260 & 260 & 260 & 260 \\
\hline L-Cystine & 3 & 3 & 3 & 3 & 3 & 3 \\
\hline Maize starch & 294 & - & - & - & - & - \\
\hline Maltodextrin & 35 & 160 & 160 & 160 & 160 & 160 \\
\hline Sucrose & 300 & 89 & 89 & 89 & 89 & 89 \\
\hline Soyabean oil & 25 & 35 & 35 & 35 & 35 & 35 \\
\hline Lard oil & 20 & 318 & 318 & 318 & 318 & 318 \\
\hline Cellulose & 50 & 62 & 62 & 62 & 62 & 62 \\
\hline Mineral mix & 13 & 13 & 13 & 13 & 13 & 13 \\
\hline Calcium hydrogen phosphate & 17 & 17 & 17 & 17 & 17 & 17 \\
\hline Calcium carbonate & 7 & 7 & 7 & 7 & 7 & 7 \\
\hline Citric acid tripotassium salt & 21 & 21 & 21 & 21 & 21 & 21 \\
\hline Vitamin mix & 13 & 13 & 13 & 13 & 13 & 13 \\
\hline Choline & 2 & 2 & 2 & 2 & 2 & 2 \\
\hline \multicolumn{7}{|l|}{ Extra additions ( $\mathrm{g} / \mathrm{kg})$} \\
\hline Sodium acetate & _- & _- & 50 & _- & - & 30 \\
\hline Sodium propionate & - & - & - & 50 & - & 10 \\
\hline Sodium butyrate & - & - & - & - & 50 & 10 \\
\hline Energy $(\mathrm{kJ} / \mathrm{g})$ & $15 \cdot 60$ & 22.08 & 22.79 & 22.79 & 22.79 & 22.79 \\
\hline $\mathrm{Na}$ content $(\mathrm{g} / \mathrm{kg})$ & $9 \cdot 6$ & $13 \cdot 2$ & $27 \cdot 2$ & $25 \cdot 1$ & $23 \cdot 6$ & $26 \cdot 1$ \\
\hline
\end{tabular}


Use of Laboratory Animals of National Administration Regulations on Laboratory Animals of China. All experimental protocols were approved by the Committee on the Ethics of Animal Experiments of the Academy of Military Medical Sciences.

\section{Quantitative analysis of mRNA expression}

Total RNA was extracted from mouse epididymal fat using TRIzol Reagent (catalogue no. 15596-026; Invitrogen), and complementary DNA was prepared from the total RNA using the SuperScriptTM III First-Strand Synthesis System for RT-PCR (catalogue no. 18080-051; Invitrogen), according to the procedures provided by manufacturer. The mRNA levels of adiponectin, resistin, DNMT1, DNMT3a, DNMT3b and the methyl-CpG-binding domain-based protein $2(M B D 2)$ in fat were measured by the real-time quantitative RT-PCR using an ABI PRISM 7300 sequence detection system (Applied Biosystems). The oligonucleotide primers for the target genes were designed with the assistance of PrimerExpress software from the gene sequence obtained from Ensembl Genome Browser (www. ensembl.org). The oligonucleotide primer pairs used are shown in the online Supplementary Table S1. The co-amplification of mouse $\beta$-actin mRNA, an invariant internal control, was performed in all of the samples. The assays were performed in triplicate, and the results were normalised to the $\beta$-actin mRNA levels by using the $2^{-\Delta C_{T}}$ method.

\section{Bisulphite conversion and sequencing}

The examined promoter regions of adiponectin and resistin are listed in Fig. $1^{(16,34,35)}$. Specifically, the adiponectin promoter region examined spans NTS 23145384-23146082 and includes six CpG sites within NTS -1162 to -494 (with respect to the TSS). The resistin promoter region examined spans NTS 3654370-3655769 and includes eighteen CpG sites within NTS -1450 to -113 (with respect to the TSS). DNA methylation of the selected site-specific CpG sites from these two adipokines was determined by bisulphite sequencing. Briefly, DNA Purification Kit (catalogue no. DN 1008; BioFuture Group Inc.) was used to isolate and purify DNA from the adipose tissue. Bisulphite conversion was performed using the MethylampTM DNA Modification Kit (catalogue no. P-1001; EpiGentek Group Inc.). Converted DNA was used fresh or it was stored at $-20^{\circ} \mathrm{C}$. Converted DNA was amplified by nested PCR, and the PCR products were sequenced directly. The methylation fraction was calculated from the amplitude of cytosine and thymine within each $\mathrm{CpG}$ dinucleotide $(\mathrm{C} /(\mathrm{C}+\mathrm{T}))$. The assays were performed in triplicate. The primers used and annealing temperature are shown in the online Supplementary Table S2.

\section{Chromatin immunoprecipitation}

Many promoter-associated proteins have been shown to be involved in the methylation regulation of gene transcription. We targeted the key enzymes for which specific antibodies are available. The chromatin immunoprecipitation (ChIP) studies were performed using either prepared EpiQuikTM Kits (EpiGentek Group Inc.) - that is, the Tissue MBD2 ChIP Kit (catalogue no. P-2018) or the general ChIP Kit (EpiGentek (a)

$\left.\begin{array}{l}-1162 \text { ttggccagcctttgcctgggagcagtctagctctgagtgtcttattggagc } \\ -1111 \text { agctgctggcatccagagttcttttggattcacg }{ }^{6} \text { atttaattcaaaagcttt } \\ -1048 \text { gtgctcccg }^{5} \text { agaatcagctctggtctttcaaaaataagatgtgagtccg } \\ -999 \mathrm{ccg}^{3} \text { agaggctcccaaggtattgccttg } \\ \ldots \ldots \ldots \\ -641 \text { catggtcttttctcagtatgggatccg } \\ -592 \text { gtctagcaagtggagcacacctt } \\ -536 \text { acaccaaaagaaggcagcg } \\ -490 \text { gatcccatggaaattactgtgggtgctg }\end{array}\right]$ A2

(b)

-1450 aaaggaagttctcacctcaggtctccagcaatgcctgggaatggaga -1402 cccccaaagcagctgtgtactatgttggcg ${ }^{18}$ atggccactaaagtcg ${ }^{17}$ -1356 catgaacacagaagccaggtgggaggaagagcacatcacctgaat -1311 ccacttatgctgaagaggaaagagacaaatcttgcacg ${ }^{16}$ gggtggg -1266 ggtggggaggcttagttcagttagagtgcctggcaggcacaaagtcc -1219 tgtgctcaagtcctcg ${ }^{15}$ aagtacagaaaccatgcacagtggtgcg ${ }^{14}$ -1175 tccctgccaccccagcatcg ${ }^{13}$ agaatgcatagcttacaaaagagc ..........

-905 cctctgctaatctcaatttgtcctatctagttaaaagacatcg ${ }^{12}$ aggcc -856 agtgtaacagcttcagagccg ${ }^{11}$ gacagcctgacg10ttaacaccc -814 aggatttgcatagccacctccagttgtcctcacctccg9tatgcacatc -766 acactgtggcg ${ }^{8}$ tgcacgcacttacacccacacg ${ }^{7}$ tgtcacg6cac -723 atgcaataatgactaaataatgttttaaagacagagaagataaggca -675 gattctattgagtcattcacacacataaatattctgtatgtgggccg ${ }^{5} \mathrm{ga}$ ..........

-414 gctcttgcttagccccacccccacccc ${ }^{4}$ cccccagagagggget -370 gggtctctagctctgtggtagcaagggagcagttgactagattttagct -321 ggtagagcttgcctacactgtatatccccg ${ }^{3} \mathrm{ccaaacatagaaacat}$ -275 acatgacagctgtggtcacacagggaccg ${ }^{2}$ tgtctcagtttgcatctc -228 cagccctacaggtgaagtcttggctcctagccttgcccctcccaccat -180 ggtccctggtgttatctccagacaacg ${ }^{1}$ tcctgagaagacaatccttct -132 aatgtaggaagggctgagca

Fig. 1. Regions of the mouse adiponectin and resistin promoters. The CG dinucleotides, assigned to each of the analysed CG, are marked and numbered on the top right. (a) The adiponectin promoter sequence with three regions spanning nucleotides -1162 to -494 . (b) The resistin promoter sequence with two regions spanning nucleotides -1450 to -113 .

catalogue no. P-2003) with specific antibodies for DNMT (1, 3a and 3b) (catalogue no. ab13537, ab2850 and ab2851). Briefly, the procedure was performed as follows. Adipose tissue (20 mg) was cross-linked with $1 \%$ formaldehyde for $20 \mathrm{~min}$ and then homogenised. The homogenate was sonicated for four pulses of $15 \mathrm{~s}$ each at level 2 using the microtip probe of a Branson Digital Sonifier (Model 450; Branson UI-transonics Corporation), followed by a 40-s interval on ice between each pulse to generate fragments of genomic DNA ranging from 200 to $1000 \mathrm{bp}$ in length. For the ChIP assays, equivalent amounts of treated chromatin were added to microwells containing immobilised antibodies for the targeted protein or a normal mouse IgG antibody negative control. In addition, a small portion of treated chromatin equivalent to $5 \%$ of the extracted genomic DNA was used as the Input DNA to calculate the enrichment of the targeted proteins. After incubation for $90 \mathrm{~min}$ at $65^{\circ} \mathrm{C}$ to reverse the cross-links and elute the DNA, fast-spin columns were used for DNA purification.

The purified DNA was used for a real-time PCR analysis of the adiponectin and resistin promoters. The sequence data for these genes are available from NCBI (http://www.ncbi.nlm.nih.gov). Specific primer pairs were designed (online Supplementary 
Table S3). The PCR conditions were as follows: $95^{\circ} \mathrm{C}$ for $10 \mathrm{~min}$, forty cycles of $95^{\circ} \mathrm{C}$ for $30 \mathrm{~s}, 60^{\circ} \mathrm{C}$ for $30 \mathrm{~s}$ and $75^{\circ} \mathrm{C}$ for $45 \mathrm{~s}$, and finally $7 \mathrm{~min}$ at $75^{\circ} \mathrm{C}$ and $2 \mathrm{~min}$ at $4^{\circ} \mathrm{C}$. A melting curve was generated from 70 to $95^{\circ} \mathrm{C}$, and the signal was read every $0.5^{\circ} \mathrm{C}$ for $5 \mathrm{~s}$. A co-amplification of mouse glyceraldehyde-3-phosphate dehydrogenase (GAPDH) mRNA was performed in all of the samples, including the ChIP and Input DNA samples. Each sample was normalised to its respective Input PCR product using the $2^{-\Delta C_{T}}$ method. The data were then expressed as the ratio of ChIP:Input, which reflects the quantity of the methylation regulatory proteins associated with the targeted gene promoter.

\section{Statistical analysis}

One-way ANOVA was performed to compare means in different groups for normal distribution data using SPSS version 13.0 for Windows; as with non-normal distribution, the differences between different groups were determined by applying the Mann-Whitney $U$ test and the Wilcoxon signed-rank test. Significant differences between the lean control group and the DIO group were assessed using Dunnett's T3 test, and comparisons between the DIO group and groups feeding different SCFA were determined by the $\mathrm{S}-\mathrm{N}-\mathrm{K}$ test. All values were expressed as means and standard deviations, and $P<0.05$ was considered to be statistically significant.

\section{Results}

\section{Effects of SCFA on body weight of obese mice}

After 16 weeks of feeding, the body weight of mice in the five HFD diet groups was significantly higher than that in the control diet group $(P<0.05)$. If the five HFD diet groups were compared, dietary supplementation of acetate, propionate, butyrate or their admixture significantly suppressed the HFD-induced weight gain $(P<0.05)$ (Fig. 2). Dietary supplementation of acetate, propionate, butyrate or their admixture to the HFD did

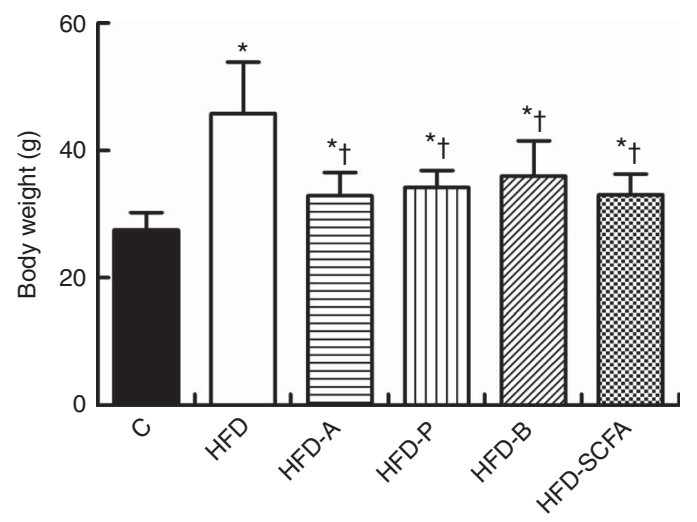

Fig. 2. SCFA inhibit body weight gain in high-fat-diet-induced obese mice. C57BL/6J male mice, 3-4 weeks old, were fed a high-fat diet (HFD) diet and four SCFA-containing HFD diets HFD with acetate (HFD-A), HFD with propionate (HFD-P), HFD with butyrate (HFD-B) and HFD with their admixture (HFD-SCFA), with a low-fat diet as control (C). Body weight changes were detected at the end of 16 weeks' feeding $(n 10)$ in each group. * Compared with the lean control group $(P<0.05)$; $†$ compared with the HFD group $(P<0.05)$. not change the intakes of both food and energy since 8 weeks after the feeding intervention $(P>0.05)$, which has been demonstrated previously in our study ${ }^{(6)}$.

\section{SCFA affect gene expressions of adiponectin and resistin in high-fat-diet-induced obese mice}

As shown in Fig. 3, compared with the control diet group, the mRNA expression levels of adiponectin and resistin were lower in the HFD groups $(P<0 \cdot 05)$. Dietary supplementation of all three SCFA or their mixture in the HFD significantly increased the mRNA levels of adiponectin and resistin $(P<0.05)$.

\section{SCFA affect gene expressions of DNA methyltransferases and methyl-CpG-binding domain protein 2 in high-fat- diet-induced obese mice}

The mRNA expressions of three types of $D N M T$ and $M B D 2$ were significantly higher in the HFD group compared with those in the control diet group $(P<0 \cdot 05)$. Dietary supplementation of all three SCFA or their mixture significantly reduced mRNA
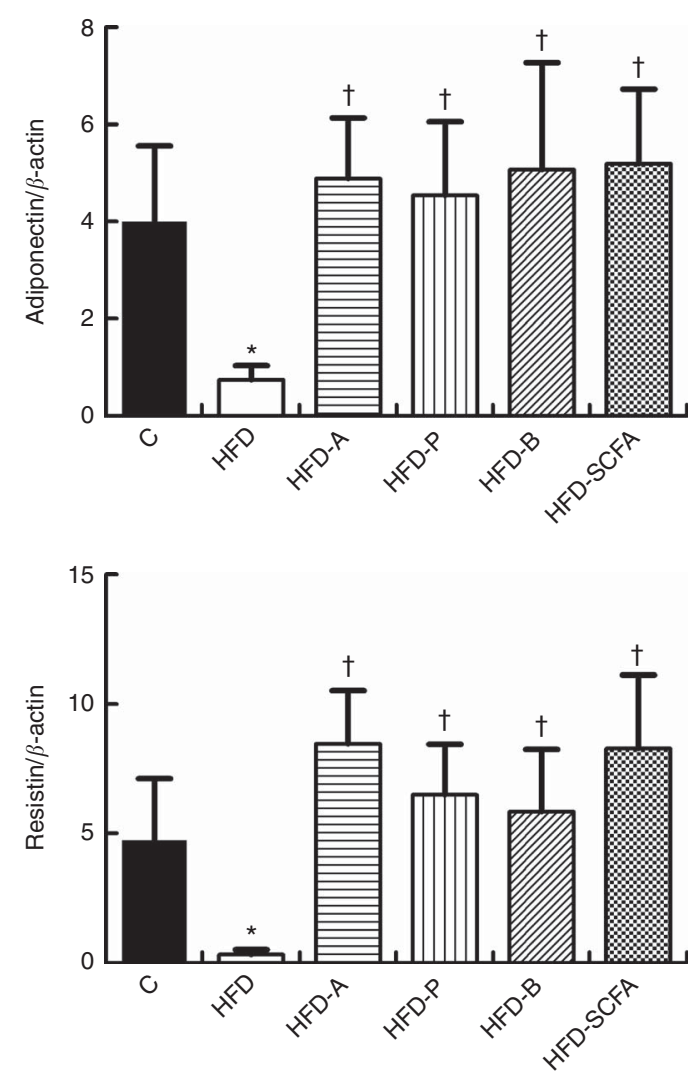

Fig. 3. Effects of SCFA on the mRNA expression of adiponectin and resistin in high-fat-diet-induced obese mice. Male C57BL/6J mice, 3-4 weeks old, were fed a high-fat diet (HFD) diet and four SCFA-containing HFD diets HFD with acetate (HFD-A), HFD with propionate (HFD-P), HFD with butyrate (HFD-B) and HFD with their admixture (HFD-SCFA) for 3 months, with a low-fat diet as control (C). RT-PCR was used to measure the mRNA levels of adiponectin and resistin in epididymal fat $(n 10)$ in each group. The data were normalised to $\beta$-actin mRNA levels using the $2^{-\Delta C_{T}}$ method. Values are means and standard deviations. * Compared with the $\mathrm{C}$ diet $(P<0.05)$; † compared with the HFD group $(P<0.05)$. 

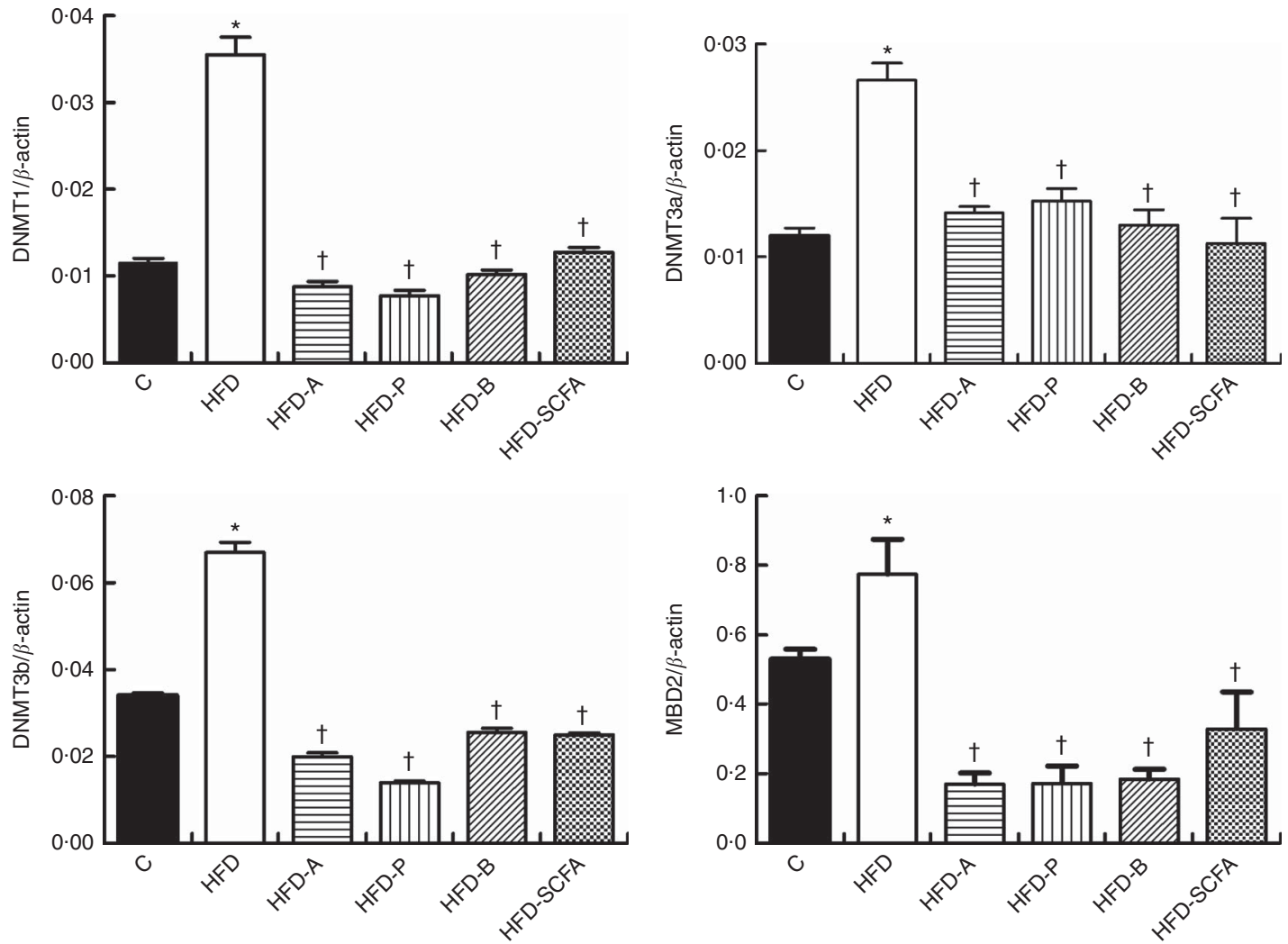

Fig. 4. Effects of SCFA on the mRNA expression of DNA methyltransferases (DNMT) and methyl-CpG-binding protein 2 (MBD2) in high-fat-diet-induced obese mice. Male C57BL/6J mice, 3-weeks old, were fed a high-fat diet (HFD) diet and four SCFA-containing HFD diets HFD with acetate (HFD-A), HFD with propionate (HFD-P), HFD with butyrate (HFD-B) and HFD with their admixture (HFD-SCFA) for 3 months, with a low-fat diet as control (C). RT-PCR was used to measure the mRNA levels of DNMT1, DNMT3a, DNMT3b and MBD2 in epididymal fat $(n 10)$ in each group. The data were normalised to $\beta$-actin mRNA levels using the $2^{-\Delta C_{T}}$ method. Values are means and standard deviations. * Compared with the $\mathrm{C}$ diet $(P<0.05)$; $\dagger$ compared with the HFD group $(P<0.05)$

expression levels of three types of DNMT and MBD2 $(P<0.05)$ (Fig. 4).

\section{SCFA affect CPG methylation in promoters of adiponectin and resistin in high-fat-diet-induced obese mice}

For the six CpG sites in the promoter region of adiponectin, the methylation fractions of sites $1-4$ in the HFD-fed mice were increased, and these increased methylation levels in the HFD-fed mice were also reversed by dietary SCFA supplementation $(P<0.05)$ (Table 2$)$.

Among the observed eighteen $\mathrm{CpG}$ sites of the methylation fractions at the resistin promoter, the higher methylation fractions of sites 5,9,11, 13 and 14 in the HFD-fed mice was found $(P<0 \cdot 05)$. Dietary SCFA supplementation reversed the changes at some of these sites, especially acetate, having the greatest effects in sites $3,5,9$ and $10 \quad(P<0.05)$ (Table 2).

\section{SCFA change DNA methyltransferases and methyl-CPG-} binding domain protein 2 at promoters of the adiponectin and resistin in obesity

As shown in Fig. 5, the HFD feeding increased the binding of DNMT1, DNMT3a, DNMT3b and MBD2 to promoters of adiponectin and resistin, and their bindings were reduced by dietary SCFA supplementation $(P<0 \cdot 05)$.

\section{Discussion}

At present, adipose tissue is also considered to be an endocrine gland that produces bioactive adipokines, such as leptin, adiponectin, resistin, apelin, visfatin and cytokines: TNF- $\alpha$ and IL- ${ }^{(22)}$. Among them, adiponectin possesses the characteristics of anti-diabetic, anti-inflammatory and anti-atherogenic activity, and has a key role in glucose and fat metabolism ${ }^{(9)}$. Meanwhile, resistin regulates whole-body insulin sensitivity ${ }^{(23)}$. It was reported that altered adipokine secretion was related to body fat accumulation and associated with the disturbance in the lipid and glucose metabolism and adipogenesis under a HFD ${ }^{(24)}$. Usually, the reduced adiponectin expression indicates an abnormality in adipose tissue function ${ }^{(25)}$. Up to now, there remains inconsistency in resistin expression, but in several different models of obesity, including the ob/ob, db/db, tub/tub and $\mathrm{KKA}(\mathrm{y})$ mice, resistin expression is significantly decreased in the white adipose tissue when compared with their lean counterparts $^{(26)}$. Our results also showed decreased mRNA levels of adiponectin and resistin in adipose tissue of DIO male mice. It is noteworthy that that female mice were not included 
Table 2. Quantitative methylation analysis in promoters of adiponectin and resistinł (Mean values and standard deviations)

\begin{tabular}{|c|c|c|c|c|c|c|c|c|c|c|c|c|c|c|c|c|c|c|c|}
\hline \multirow[b]{2}{*}{ Genes } & \multirow[b]{2}{*}{ Diets } & \multicolumn{18}{|c|}{ CpG sites } \\
\hline & & Mean & SD & Mean & SD & Mean & SD & Mean & SD & Mean & SD & Mean & SD & Mean & SD & Mean & SD & Mean & SD \\
\hline \multicolumn{20}{|c|}{ Adiponectin } \\
\hline & & \multicolumn{2}{|c|}{ CG1 } & \multicolumn{2}{|c|}{ CG2 } & \multicolumn{2}{|c|}{ CG3 } & \multicolumn{2}{|c|}{ CG4 } & \multicolumn{2}{|c|}{ CG5 } & \multicolumn{2}{|c|}{ CG6 } & \multicolumn{2}{|c|}{ Average } & & & & \\
\hline & C & 0.84 & 0.03 & 0.82 & 0.03 & 0.81 & 0.05 & 0.90 & 0.03 & 0.89 & 0.06 & 0.82 & 0.05 & 0.85 & 0.04 & & & & \\
\hline & HFD & $0.91^{*}$ & 0.02 & $0.86^{*}$ & 0.02 & $0.85^{\star}$ & 0.02 & $0.95^{*}$ & 0.02 & 0.89 & 0.04 & 0.84 & 0.08 & 0.88 & 0.03 & & & & \\
\hline & HFD-A & $0.87 \dagger$ & 0.01 & $0.82 \dagger$ & 0.01 & $0.80 \dagger$ & 0.03 & $0.90 \dagger$ & 0.02 & 0.88 & 0.04 & 0.84 & 0.06 & 0.85 & 0.03 & & & & \\
\hline & HFD-P & $0.86 \dagger$ & 0.02 & $0.81 \dagger$ & 0.02 & 0.81 & 0.05 & $0.88 \dagger$ & 0.03 & 0.88 & 0.05 & 0.79 & 0.09 & 0.84 & 0.04 & & & & \\
\hline & HFD-B & $0.87 \dagger$ & 0.03 & $0.82 \dagger$ & 0.04 & 0.82 & 0.04 & $0.91 \dagger$ & 0.03 & 0.89 & 0.04 & 0.83 & 0.04 & 0.86 & 0.04 & & & & \\
\hline & HFD-SCFA & $0.88 \dagger$ & 0.02 & $0.84 \dagger$ & 0.02 & $0.79 \dagger$ & 0.05 & $0.89 \dagger$ & 0.04 & 0.90 & 0.02 & 0.82 & 0.03 & 0.85 & 0.03 & & & & \\
\hline \multirow[t]{15}{*}{ Resistin } & & & & & & & & & & & & & & \multirow{2}{*}{\multicolumn{2}{|c|}{ CG9 }} & \multirow{2}{*}{\multicolumn{2}{|c|}{ CG10 }} & & \\
\hline & & \multicolumn{2}{|c|}{ CG3 } & \multicolumn{2}{|c|}{ CG4 } & \multicolumn{2}{|c|}{ CG5 } & \multicolumn{2}{|c|}{ CG6 } & \multicolumn{2}{|c|}{ CG7 } & \multicolumn{2}{|c|}{ CG8 } & & & & & \multicolumn{2}{|c|}{ CG11 } \\
\hline & C & 0.73 & 0.03 & 0.78 & 0.03 & 0.67 & 0.06 & 0.74 & 0.04 & 0.74 & 0.04 & 0.76 & 0.04 & 0.68 & 0.06 & 0.83 & 0.04 & 0.81 & 0.04 \\
\hline & HFD & 0.74 & 0.02 & 0.79 & 0.03 & $0.72^{\star}$ & 0.05 & 0.77 & 0.03 & 0.76 & 0.02 & 0.77 & 0.03 & $0.75^{\star}$ & 0.04 & 0.86 & 0.03 & $0.84^{\star}$ & 0.02 \\
\hline & HFD-A & $0.70 \dagger$ & 0.04 & 0.77 & 0.04 & $0.64 \dagger$ & 0.02 & $0.73 \dagger$ & 0.03 & $0.68 \dagger$ & 0.04 & $0.73 \dagger$ & 0.06 & $0.67 \dagger$ & 0.04 & $0.73 \dagger$ & 0.17 & $0.76 \dagger$ & 0.03 \\
\hline & HFD-P & 0.72 & 0.05 & 0.78 & 0.04 & $0.65 \dagger$ & 0.04 & 0.75 & 0.05 & $0.69 \dagger$ & 0.04 & 0.74 & 0.06 & $0.68 \dagger$ & 0.04 & 0.78 & 0.09 & $0.77 \dagger$ & 0.06 \\
\hline & HFD-B & 0.71 & 0.04 & 0.78 & 0.04 & $0.66 \dagger$ & 0.04 & 0.74 & 0.05 & $0.70 \dagger$ & 0.05 & 0.74 & 0.03 & $0.70 \dagger$ & 0.05 & 0.81 & 0.03 & $0.79 \dagger$ & 0.03 \\
\hline & HFD-SCFA & 0.73 & 0.04 & 0.81 & 0.02 & $0.67 \dagger$ & 0.03 & 0.76 & 0.04 & $0.72 \dagger$ & 0.03 & 0.76 & 0.03 & $0.69 \dagger$ & 0.03 & 0.82 & 0.03 & $0.80 \dagger$ & 0.04 \\
\hline & & \multicolumn{2}{|c|}{ CG12 } & \multicolumn{2}{|c|}{ CG13 } & \multicolumn{2}{|c|}{ CG14 } & \multicolumn{2}{|c|}{ CG15 } & \multicolumn{2}{|c|}{ CG16 } & \multicolumn{2}{|c|}{ CG17 } & $\mathrm{CG}$ & & Aver & age & & \\
\hline & C & 0.76 & 0.04 & 0.64 & 0.03 & 0.74 & 0.03 & 0.54 & 0.04 & 0.79 & 0.02 & 0.83 & 0.09 & 0.93 & 0.02 & 0.75 & 0.04 & & \\
\hline & HFD & 0.78 & 0.04 & $0.73^{\star}$ & 0.03 & $0.81^{*}$ & 0.03 & 0.64 & 0.05 & 0.81 & 0.02 & 0.86 & 0.02 & 0.93 & 0.01 & $0.79^{*}$ & 0.03 & & \\
\hline & HFD-A & 0.78 & 0.03 & 0.70 & 0.06 & 0.77 & 0.03 & 0.60 & 0.08 & 0.80 & 0.03 & 0.88 & 0.02 & 0.93 & 0.01 & 0.74 & 0.05 & & \\
\hline & HFD-P & 0.75 & 0.03 & $0.64 \dagger$ & 0.06 & $0.75 \dagger$ & 0.04 & 0.55 & 0.03 & 0.79 & 0.02 & 0.88 & 0.02 & 0.93 & 0.01 & 0.74 & 0.04 & & \\
\hline & HFD-B & $0.73 \dagger$ & 0.04 & $0.66 \dagger$ & 0.05 & $0.75 \dagger$ & 0.07 & 0.56 & 0.06 & 0.79 & 0.02 & 0.86 & 0.02 & 0.94 & 0.01 & 0.74 & 0.04 & & \\
\hline & HFD-SCFA & 0.76 & 0.05 & $0.65 \dagger$ & 0.08 & $0.76 \dagger$ & 0.04 & 0.57 & 0.08 & 0.78 & 0.03 & 0.87 & 0.02 & 0.94 & 0.01 & 0.76 & 0.04 & & \\
\hline
\end{tabular}

C, control; HFD, high-fat diet; HFD-A, HFD with acetate; HFD-P, HFD with propionate; HFD-B, HFD with butyrate; HFD-SCFA, HFD with admixture of acetate, butyrate and propionate. * Compared with the $\mathrm{C}$ group $(P<0.05)$.

$\dagger$ Compared with the HFD $(P<0.05) . n 10$ in each group.

¥ The methylation fraction was calculated from the amplitude of cytosine and thymine within each $\mathrm{CpG}$ dinucleotide, $\mathrm{C} /(\mathrm{C}+\mathrm{T})$. The assays were performed in triplicate.

in our study, which could be a limitation. We used male mice herein because the male rodents are introduced as 'gold standards' for studying diet-induced obesity ${ }^{(21)}$. In addition, expression levels of the resistin and adiponectin genes are sexspecific, which easily change owing to endogenous and exogenous factors in females ${ }^{(23,27)}$. The effects of dietary SCFA supplementation on expressions of adiponectin and resistin in female DIO mice need to be elucidated in the future.

Nevertheless, the increased mRNA levels of adiponectin and resistin in DIO mice were observed when supplemented with dietary acetate, propionate, butyrate or their admixture in our study. Similarly, it is also reported that short-term oral administration of SCFA can alleviate diet-induced obesity and insulin resistance in mice through activation of the adiponectin-mediated pathway and stimulation of mitochondrial function in the skeletal muscle ${ }^{\text {(28) }}$. However, at present, the effect of exogenous SCFA on resistin expression has not yet been reported. Our results markedly indicate that SCFA may improve expressions of these two adipokines, and this might be helpful for obesity prevention.

It has been previously shown that epigenetic modification is responsible for chromatin structure and stability, and that DNA methylation is one of the key players governing gene expression independent of modulating expressions of transcription factors. Thus, epigenetic modification may be an important contributor to many chronic diseases including obesity ${ }^{(29,30)}$. DNA methylation is the only known modification that targets the DNA itself. Moreover, aberrant DNA methylation can silence or activate gene expression patterns $^{(31)}$. Single CpG site methylation in the region of a gene's promoter can participate in the regulation of transcription, especially when it lies in a relevant transcription factor-binding site, and could be associated with diseases ${ }^{(32,33)}$. Previous studies using animal models and pharmacological approaches have also shown that DNA methylation of a particular promoter region (relatively close to the transcription start site) suppresses the adiponectin expression $^{(16,32,34)}$. Diet-induced variability of the resistin gene transcript level and methylation profile in rats was also reported by analysing the CpG island located from -945 to -1193 upstream of the first exon of the resistin gene ${ }^{(35)}$. In this study, methylation levels of six $\mathrm{CpG}$ and eighteen $\mathrm{CpG}$ in the regions spanning the first exon and the promoter ( $1.5 \mathrm{~kb}$ upstream of TSS) of adiponectin and resistin were examined, respectively. The results have shown that the higher methylation fractions of four CpG sites (CG1, CG2, CG3 and CG4) in the adiponectin gene and six CpG sites (CG5, CG7, CG9, CG11, CG13 and CG14) in the resistin gene in obesity were obviously reversed by dietary SCFA supplementation. This suggested that SCFA regulate expression levels of adiponectin and resistin by epigenetic modification, which may result in phenotypic changes including body weight.

The results have also presented that $\mathrm{CpG}$ methylation in the special sites, not all the CPG sites, significantly changed in HFD supplemented with SCFA. The heterogeneity in nearby CpG sites may be associated with the site-specific DNA methylation differently mediated by DNA-binding transcription factors, DNA-binding methyltransferases and chromatin modifiers (ubiquitylation, acetylation and propionylation) ${ }^{(36)}$. Butyrate is well known for its epigenetic activity as a HDACi, and activities of HDAC and methyl CpG-binding proteins may influence DNA methylation ${ }^{(37,38)}$. Moreover, the research by Benjamin \& Jost ${ }^{(39)}$ provided the evidence for an alternative regulating mechanism to histone deacetylation in executing methylation-directed gene expression by SCFA. 

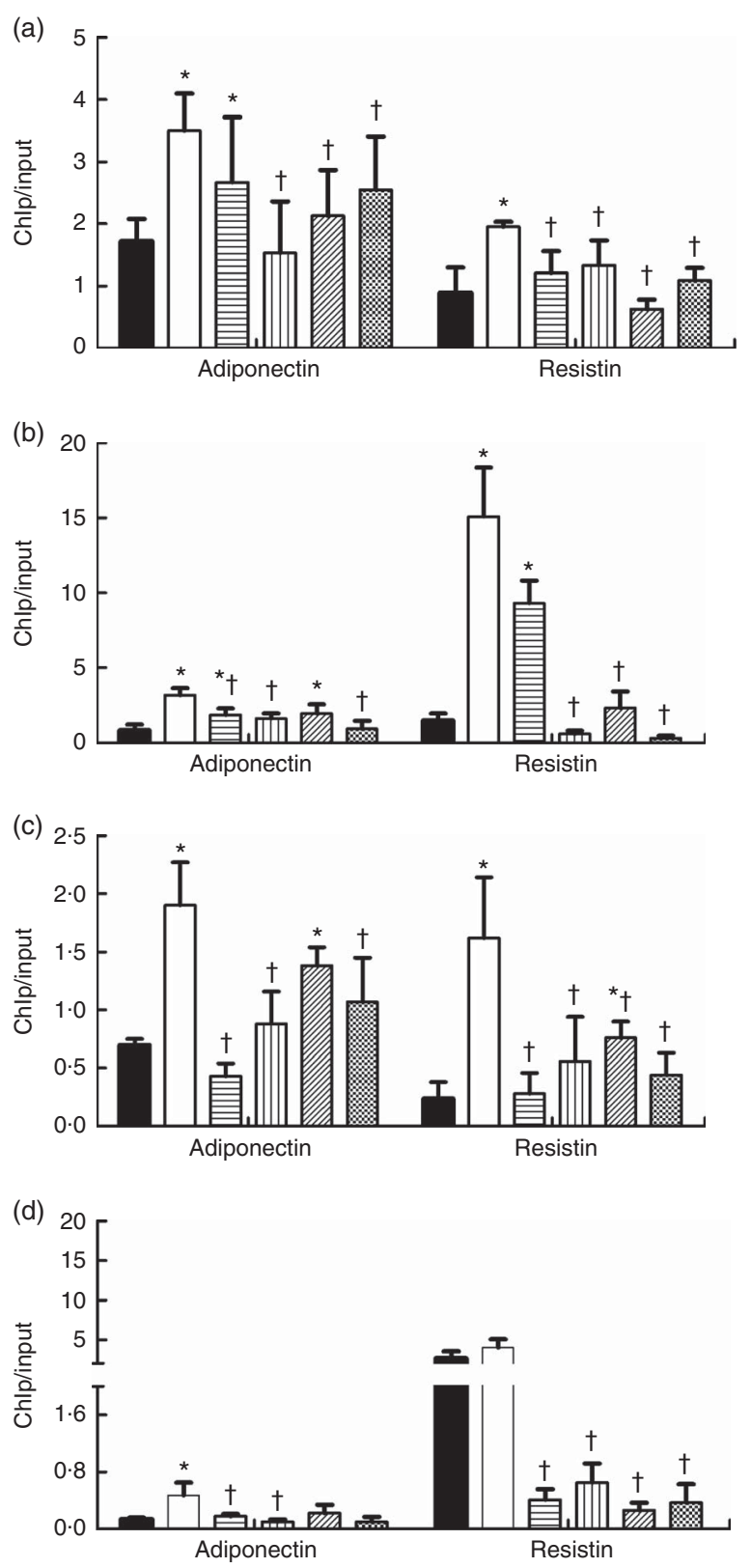

Fig. 5. Changes in DNA methyltransferases (DNMT) and methyl-CpG-binding protein 2 (MBD2) binding at the promoters of adiponectin and resistin in highfat-diet (HFD)-induced obese mice. Genomic DNA was purified from mouse epididymal fat and was immunoprecipitated with antibodies specific for (a) DNMT1, (b) DNMT3a, (c) DNMT3b and (d) MBD2. The immunoprecipitated DNA was used in PCR reactions to detect the promoter's presence of adiponectin and resistin. Normal mouse IgG was used as a negative control. The ratio of the PCR signal from the protein chromatin immunoprecipitation (ChIP) DNA: signal from the total genomic DNA (input) is plotted as an estimation of the protein levels $(n 8)$ in each group. Values are means and standard deviations. $\square$, Control (C); $\square$, HFD; 目, HFD with acetate; 向, HFD with propionate; $\mathbb{Z}$, HFD with butyrate; $\mathbb{R}$, HFD with their admixture. * Compared with the $\mathrm{C}$ diet $(P<0.05)$; $†$ compared with the HFD group $(P<0.05)$.

Addition of methyl groups to the 5 ' position on cytosine residues in DNA is catalysed by the family of DNMT proteins, including DNMT1, DNMT3a and DNMT3b. DNMT1 plays a major role in the maintenance of methylation patterns, exhibiting a higher level of activity on hemi-methylated compared with unmethylated DNA, whereas de novo methylation is catalysed by DNMT3a and DNMT3 $b^{(40,41)}$. Furthermore, the MBD family proteins specifically bind double-stranded and methylated DNA to regulate gene transcription, which makes them useful for DNA methylation analysis ${ }^{(42)}$. In $\mathrm{db} / \mathrm{db}$ mice, the hypoadiponectinaemia was ameliorated by the DNMT inhibitor RG108, which reduced DNA methylation level at the gene promoter, consequently alleviating metabolic dysregulation ${ }^{(33)}$. In the current study, we found that transcriptional expression levels of adipose $M B D 2$ and DNMT were increased in the DIO mice, but reduced after dietary supplementation with SCFA. Moreover, the HFD feeding increased bindings of DNMT1, 3a, $3 \mathrm{~b}$ and MBD2 to promoters of adiponectin and resistin, and their bindings were prohibited by dietary SCFA supplementation. Previous studies have addressed that once a gene promoter has been demethylated the gene recovers its capacity to be transcribed ${ }^{(43)}$. Thus, the effects of SCFA on mRNA expressions of the adiponectin and resistin may be attributable to the reduction in DNA methylation at gene promoters, together with decreased bindings of MBD2 and DNMT to gene promoters. To our best knowledge, this is the first report to clarify the specific DNMT subtypes and MBD2 that were responsible for DNA methylation of the adiponectin and resistin genes and associated transcriptional expressions.

Taken together, dietary supplementation of acetate, propionate, butyrate or their admixture prevented the body weight gain in the DIO male mice, and this may be associated with their regulation on adiponectin and resistin expressions. Specifically, reductions in the adiponectin and resistin expressions were reversed to normal ranges by dietary SCFA supplementation in DIO male mice, which may probably be mediated by modification of DNA methylation showing lowered expressions of DNA methylation-related enzymes (DNMT1, DNMT3a and DNMT3b) and MBD2, as well as their decreased bindings to the gene promoters.

\section{Acknowledgements}

This work was supported by the National Natural Science Foundation of China (no. 81670775) (K. Q.) and the Research Funds of Profession Quota Budjet from Beijing Municipal Science and Technology Commission (2016-bjsekyjs-2 to K. Q.).

C. F. and K. Q. designed the research. Y. L. and X. F. carried out the animal study and performed the experiment. A. L., R. W. and P. L. analysed the data. Y. L. assisted C. F. in preparing the manuscript. K. Q. helped to review the manuscript. All authors have read and approved the final manuscript.

The authors declare that there are no conflicts of interest.

\section{Supplementary material}

For supplementary material/s referred to in this article, please visit https://doi.org/10.1017/S0007114518001526

\section{References}

1. Kondo T, Kishi M, Fushimi T, et al. (2009) Vinegar intake reduces body weight, body fat mass, and serum triglyceride levels in obese Japanese subjects. Biosci Biotechnol Biochem 73, 1837-1843. 
2. Chambers ES, Viardot A, Psichas A, et al. (2015) Effects of targeted delivery of propionate to the human colon on appetite regulation, body weight maintenance and adiposity in overweight adults. Gut 64, 1744-1754.

3. den Besten G, Bleeker A, Gerding A, et al. (2015) Short-chain fatty acids protect against high-fat diet-induced obesity via a PPAR $\gamma$-dependent switch from lipogenesis to fat oxidation. Diabetes 64, 2398-2408.

4. Kasubuchi M, Hasegawa S, Hiramatsu T, et al. (2015) Dietary gut microbial metabolites, short-chain fatty acids, and host metabolic regulation. Nutrients 7, 2839-2849.

5. Meyre D, Froguel P, Horber FF, et al. (2014) Comment on: Valette et al. Melanocortin-4 receptor mutations and polymorphisms do not affect weight loss after bariatric surgery. PLOS ONE 2012; 7(11):E48221. PLOS ONE 9, e93324.

6. Lu Y, Fan C, Li P, et al. (2016) Short chain fatty acids prevent high-fat-diet-induced obesity in mice by regulating $G$ proteincoupled receptors and gut microbiota. Sci Rep 6, 37589.

7. Kimura I, Inoue D, Maeda T, et al. (2011) Short-chain fatty acids and ketones directly regulate sympathetic nervous system via G protein-coupled receptor 41 (GPR41). Proc Natl Acad Sci U S A 108, 8030-8035.

8. Leal Vde O \& Mafra D (2013) Adipokines in obesity. Clin Chim Acta 419, 87-94.

9. Vendrell J, Broch M, Vilarrasa N, et al. (2004) Resistin, adiponectin, ghrelin, leptin, and proinflammatory cytokines: relationships in obesity. Obes Res 12, 962-971.

10. Roumaud P \& Martin LJ (2015) Roles of leptin, adiponectin and resistin in the transcriptional regulation of steroidogenic genes contributing to decreased Leydig cells function in obesity. Horm Mol Biol Clin Investig 24, 25-45.

11. Bełtowski J (2003) Adiponectin and resistin-new hormones of white adipose tissue. Med Sci Monit 9, RA55-RA61.

12. Milagro FI, Mansego ML, De Miguel C, et al. (2013) Dietary factors, epigenetic modifications and obesity outcomes: progresses and perspectives. Mol Aspects Med 34, 782-812.

13. De Carvalho DD, You JS \& Jones PA (2010) DNA methylation and cellular reprogramming. Trends Cell Biol 20, 609-617.

14. Richmond RC, Timpson NJ \& Sørensen TI (2015) Exploring possible epigenetic mediation of early-life environmental exposures on adiposity and obesity development. Int J Epidemiol 44, 1191-1198.

15. Shen W, Wang C, Xia L, et al. (2014) Epigenetic modification of the leptin promoter in diet-induced obese mice and the effects of N-3 polyunsaturated fatty acids. Sci Rep 4, 5282.

16. Greenhill C (2015) Epigenetics: obesity-induced hypermethylation of adiponectin gene. Nat Rev Endocrinol 11, 504 .

17. Burdge GC \& Lillycrop KA (2010) Bridging the gap between epigenetics research and nutritional public health interventions. Genome Med 2, 80.

18. Lavebratt C, Almgren M \& Ekström TJ (2012) Epigenetic regulation in obesity. Int J Obes (Lond) 36, 757-765.

19. Waldecker M, Kautenburger T, Daumann H, et al. (2008) Inhibition of histone-deacetylase activity by short-chain fatty acids and some polyphenol metabolites formed in the colon. J Nutr Biochem 19, 587-593.

20. Spurling CC, Suhl JA, Boucher N, et al. (2008) The short chain fatty acid butyrate induces promoter demethylation and reactivation of RARbeta 2 in colon cancer cells. Nutrition and cancer $\mathbf{6 0}, 692-702$.

21. Hariri N \& Thibault L (2010) High-fat diet-induced obesity in animal models. Nutr Res Rev 23, 270-299.

22. Kuryszko J, Sławuta P \& Sapikowski G (2016) Secretory function of adipose tissue. Pol J Vet Sci 19, 441-446.

23. Ribot J, Rodríguez AM, Rodríguez E, et al. (2008) Adiponectin and resistin response in the onset of obesity in male and female rats. Obesity (Silver Spring) 16, 723-730.
24. Antuna-Puente B, Feve B, Fellahi S, et al. (2008) Adipokines: the missing link between insulin resistance and obesity. Diabetes Metab 34, 2-11.

25. Oliveira Md, de Ś́bio MT, Olimpio RM, et al. (2015) Triiodothyronine modulates the expression of leptin and adiponectin in 3T3-L1 adipocytes. Einstein (Sao Paulo) 13, 72-78.

26. Way JM, Görgün CZ, Tong Q, et al. (2001) Adipose tissue resistin expression is severely suppressed in obesity and stimulated by peroxisome proliferator-activated receptor gamma agonists. J Biol Chem 276, 25651-25653.

27. Stelmanska E, Kmiec Z \& Swierczynski J (2012) The genderand fat depot-specific regulation of leptin, resistin and adiponectin genes expression by progesterone in rat. $J$ Steroid Biochem Mol Biol 132, 160-167.

28. Hong J, Jia Y, Pan S, et al. (2016) Butyrate alleviates high fat diet-induced obesity through activation of adiponectinmediated pathway and stimulation of mitochondrial function in the skeletal muscle of mice. Oncotarget 7, 56071-56082.

29. Blackledge NP \& Klose R (2011) CpG island chromatin: a platform for gene regulation. Epigenetics 6, 147-152.

30. Vickers MH (2014) Early life nutrition, epigenetics and programming of later life disease. Nutrients 6, 2165-2178.

31. Weber M \& Schübeler D (2007) Genomic patterns of DNA methylation: targets and function of an epigenetic mark. Curr Opin Cell Biol 19, 273-280.

32. Kim AY, Park YJ, Pan X, et al. (2015) Obesity-induced DNA hypermethylation of the adiponectin gene mediates insulin resistance. Nat Commun 6, 7585.

33. Ehrlich S, Weiss D, Burghardt R, et al. (2010) Promoter specific DNA methylation and gene expression of POMC in acutely underweight and recovered patients with anorexia nervosa. J Psychiatr Res 44, 827-833.

34. Houde AA, Légaré C, Hould FS, et al. (2014) Cross-tissue comparisons of leptin and adiponectin: DNA methylation profiles. Adipocyte 3, 132-140.

35. Nowacka-Woszuk J, Pruszynska-Oszmalek E, Szydlowski M, et al. (2015) Diet-induced variability of the resistin gene (Retn) transcript level and methylation profile in rats. BMC Genet 16, 113.

36. Nishiyama A, Yamaguchi L \& Nakanishi M (2016) Regulation of maintenance DNA methylation via histone ubiquitylation. J Biochem 159, 9-15.

37. Puddu A, Sanguineti R, Montecucco F, et al. (2014) Evidence for the gut microbiota short-chain fatty acids as key pathophysiological molecules improving diabetes. Mediators Inflamm 2014, 162021.

38. Das B, Dobrowolski C, Shahir AM, et al. (2015) Short chain fatty acids potently induce latent HIV-1 in T-cells by activating P-TEFb and multiple histone modifications. Virology 474, 65-81.

39. Benjamin D \& Jost JP (2001) Reversal of methylation-mediated repression with short-chain fatty acids: evidence for an additional mechanism to histone deacetylation. Nucleic Acids Res 29, 3603-3610.

40. Potter C, McKay J, Groom A, et al. (2013) Influence of DNMT genotype on global and site specific DNA methylation patterns in neonates and pregnant women. PLOS ONE 8, e76506.

41. Lund K, Cole JJ, VanderKraats ND, et al. (2014) DNMT inhibitors reverse a specific signature of aberrant promoter DNA methylation and associated gene silencing in AML. Genome Biol 15, 406.

42. Heimer BW, Tam BE \& Sikes HD (2015) Characterization and directed evolution of a methyl-binding domain protein for high-sensitivity DNA methylation analysis. Protein Eng Des Sel 28, 543-551.

43. Renaudineau Y \& Youinou P (2011) Epigenetics and autoimmunity, with special emphasis on methylation. Keio J Med 60, 10-16. 